\section{Leishmaniose könnte häufiger werden \\ „Rollende Arztpraxis" für Flüchtlinge}

Hautkrankheiten gehören zu den häufigsten Anlässen ärztlicher Untersuchungen bei Migranten, sagte Prof. Dr. Esther von Stebut-Borschitz, Mainz. „Fast die Hälfte der Asylsuchenden kommt aus Ländern, in denen die kutane Leishmaniose endemisch ist, nämlich Syrien, Afghanistan, Irak und Pakistan“, so die Dermatologin. Die Protozoen werden durch Sandmücken übertragen, die Inkubationszeiten betragen Wochen bis Monate. Der Verlauf hänge vom Erreger und dem Immunstatus der Patienten ab. Rote Papeln, die sich zu Plaques oder Knoten entwickeln, ulzerieren und sich bakteriell infizieren können, seien Symptome.

(nsi)

DGIM-Kongress 2016 9.-12. April, Mannheim
Im Mai wurden die Preise für Gesundheitsnetzwerker verliehen. Der erste Preis geht an das Praxisnetz Herzogtum Lauenburg für seine „Rollende Arztpraxis“ zur Versorgung von Flüchtlingen auf dem Land. Schwachstellen in der medizinischen Versorgung von Asylbewerbern und Schutzsuchenden zu verbessern, sei das Ziel. „Der einrichtungsübergreifende Ansatz in der Versorgung von Flüchtlingen ist ebenso innovativ wie die Nutzung einer mobilen Praxis in diesem Kontext", erläutert SchaichWalch die Jurybegründung. „Es handelt sich um einen langfristigen Versorgungsansatz, der auch akut für Sondersituationen eingesetzt werden kann.“

(maw)

Kongresses für Gesundheitsnetzwerker, 3.-4. Mai 2016

\section{Fingerspitzengefühl gefragt!}

Die Behandlung von Flüchtlingen erfordert Fingerspitzengefühl in jeder Hinsicht. Denn neben sprachlichen Hürden kann es auch zu Missverständnissen kommen, die kulturell bedingt sind. So sagen Patienten zum Teil genau das Gegenteil von dem, was sie eigentlich meinen - meist aus Höflichkeit oder weil sie "Autoritätspersonen“ nicht widersprechen wollen. Wie Sie trotzdem zur richtigen Diagnose kommen, lesen Sie in unserem Schwerpunkt "Migrationsmedizin."

Claudia Daniels Redakteurin

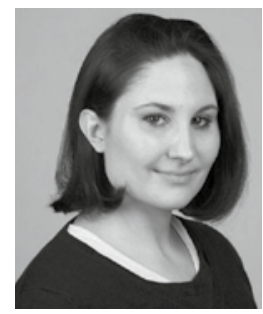

\section{Neues Impfkonzept für Asylsuchende}

Das Gesundheitsamt Reutlingen entwickelte ein Konzept, um Asylbewerbern im Landkreis den Zugang zu Schutzimpfungen zu erleichtern. Dieses Impfkonzept beinhaltet die Bereitstellung von Impfstoffen und anderen Materialien durch das Gesundheitsamt sowie die Kooperation mit der Kreisärzteschaft.

Um die ersten Erfolge zu überprüfen, wurde die Anzahl der von Ärzten aus eigener Initiative durchgeführten Impfungen mit den Impfungen verglichen, die im Rahmen des Konzepts durchgeführt wurden. Insgesamt erhielten 642 (28\%) der
2256 Asylsuchenden mindestens eine Impfung, wobei 571 (89\%) der Geimpften im Rahmen des neu entwickelten Impfkonzepts geimpft wurden. In Unterkünften, die bisher nicht in das neue Konzept eingeschlossen wurden, betrug die Impfquote nur etwa $6 \%$, in den eingeschlossenen Unterkünften bis zu 58\%.

Das Konzept zeigt damit erste Erfolge, dennoch sind weitere Verbesserungen wie z.B. eine stabsmäßige Planung und Koordinierung des Projektes notwendig. (red)

Bundesgesundheitsblatt 2016/5

\title{
Auch Flüchtlinge haben die Grippe
}

Die Daten größerer Einrichtungen und eine Umfrage unter DGIM-Mitgliedern weisen darauf hin: Migranten haben häufig dieselben akuten Erkrankungen wie die einheimische Bevölkerung: Grippe, Erkältung Windpocken. Hinzu kommen gastrointestinale Infektionskrankheiten durch Noroviren, Salmonellen, Shigellen oder Campylobacter. Probleme bereiten in Erstaufnahmeunterkünften Skabies (Krätze) und das Läuserückfallfieber. „Ärzte müssen mit allen Erkrankungen rechnen, die deutsche Patienten auch bekommen", sagt Dr. August Stich, Tropenmediziner von der Missionsärztlichen Klinik Würzburg. Und sie müssten an seltene Erkrankungen denken, z.B. aufgrund von Mangelernährung und Hypovitaminosen sowie an Hämoglobinopathien und übertragbare Erkrankungen. Hinzu kämen häufig Traumata und posttraumatische Belastungsstörungen. „Dringend notwendig sind jetzt eine individuelle medizinische Versorgung der Migranten und eine systematische Erfassung ihrer Erkrankungen und des Therapieverlaufs“, so Stich.

(nsi)

DGIM-Kongress 2016 9.-12. April, Mannheim

\section{Vormarsch der Tuberkulose}

Die Tuberkulosezahlen in Deutschland steigen. Lagen die Inzidenzen ab 2009/2010 zwischen 5,2 und 5,6 pro 100.000 Einwohner, könnte die Rate für das vergangene Jahr bei 7 pro 100.000 liegen. Bundesweit waren dem Robert Koch-Institut in Berlin 4533 Tuberkulosen im Jahr 2014 gemeldet worden, das sind circa 200 mehr als im Jahr zuvor. Der Trend nach oben setzte sich dann im letzten Jahr mit 5865 Erkrankungen fort. „Es ist klar, dass der Anstieg auch auf die aktuellen Migrationsbewegungen zurückzuführen ist“, sagte Dr. Stefan Schmiedel, Hamburg-Eppendorf. Dennoch: Flüchtlinge in Deutschland seien keine wesentliche Gefährdung für die Bevölkerung in Deutschland, das gelte auch für die Tuberkulose.

Robert Koch Institut (RKI); Epid Bulletin 10/11; 2016.

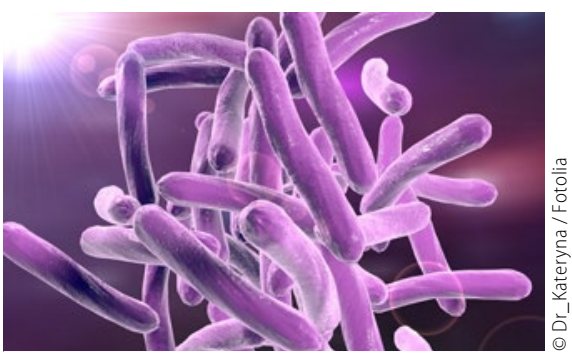

Mykobakterien sind die Erreger der Tbc. 